\title{
TWO OPERATOR FUNCTIONS WITH MONOTONE PROPERTY
}

\author{
TAKAYUKI FURUTA
}

(Communicated by Palle E. T. Jorgensen)

\begin{abstract}
This paper proves that $F(p)=\left(B^{r} A^{p} B^{r}\right)^{(1+2 r) /(p+2 r)}$ is an increasing function of $p$ for $p \geqq 1$ and $r \geqq 0$ whenever $A \geqq B \geqq 0$. This result is more precise than our previous result that $A \geqq B \geqq 0$ ensures $\left(B^{r} A^{p} B^{r}\right)^{(1+2 r) /(p+2 r)} \geqq B^{1+2 r}$ for each $p \geqq 1$ and $r \geqq 0$. We also cite three counterexamples related to Theorem 1 .
\end{abstract}

\section{Statement OF Results}

A capital letter will mean a bounded linear operator on a Hilbert space. It is well known that $A \geqq B \geqq 0$ does not always ensure $A^{2} \geqq B^{2}$ in general and that " $\geqq$ " is only a partial order on the set of positive operators, not a total ordering. But Theorem 1 ensures order-preserving inequalities on $A$ and $B$ in the case $A \geqq B \geqq 0$.

Theorem 1. If $A \geqq B \geqq 0$, then for each $r \geqq 0$ and each $p \geqq 1$,

(i) $F(p)=\left(B^{r} A^{p} B^{r}\right)^{(1+2 r) /(p+2 r)}$ is an increasing function of $p$; that is, for any set $\left\{p_{k}\right\}$ of increasing real numbers such that $p_{n} \geqq p_{n-1} \geqq \cdots \geqq p_{2} \geqq p_{1}=1$,

$$
\begin{aligned}
\left(B^{r} A^{p_{n}} B^{r}\right)^{(1+2 r) /\left(p_{n}+2 r\right)} & \geqq\left(B^{r} A^{P_{n-1}} B^{r}\right)^{(1+2 r) /\left(p_{n-1}+2 r\right)} \\
& \geqq \cdots \geqq\left(B^{r} A^{p_{2}} B^{r}\right)^{(1+2 r) /\left(p_{2}+2 r\right)} \geqq B^{r} A B^{r} \geqq B^{1+2 r} .
\end{aligned}
$$

(ii) $G(p)=\left(A^{r} B^{p} A^{r}\right)^{(1+2 r) /(p+2 r)}$ is a decreasing function of $p$; that is, for any set $\left\{p_{k}\right\}$ of increasing real numbers such that $p_{n} \geqq p_{n-1} \geqq \cdots \geqq p_{2} \geqq p_{1}=1$,

$$
\begin{aligned}
A^{1+2 r} & \geqq A^{r} B A^{r} \geqq\left(A^{r} B^{p_{2}} A^{r}\right)^{(1+2 r) /\left(p_{2}+2 r\right)} \\
& \geqq \cdots \geqq\left(A^{r} B^{p_{n-1}} B^{r}\right)^{(1+2 r) /\left(p_{n-1}+2 r\right)} \geqq\left(A^{r} B^{p_{n}} A^{r}\right)^{(1+2 r) /\left(p_{n}+2 r\right)} .
\end{aligned}
$$

Corollary 1. If $A \geqq B \geqq 0$, then for each $p \geqq 1$,

(i) $\left(B A^{p} B\right)^{3 /(p+2)} \geqq\left(B A^{p-1} B\right)^{3 /(p+1)} \geqq \cdots \geqq\left(B A^{2} B\right)^{3 / 4} \geqq B A B \geqq B^{3}$ and

(ii) $A^{3} \geqq A B A \geqq\left(A B^{2} A\right)^{3 / 4} \geqq \cdots \geqq\left(A B^{p-1} A\right)^{3 /(p+1)} \geqq\left(A B^{p} A\right)^{3 /(p+2)}$.

Received by the editors December 14, 1989.

1980 Mathematics Subject Classification (1985 Revision). Primary 47B15.

Key words and phrases. Positive operator, order-preserving inequalities. 


\section{PROOFS OF THE RESULTS}

First of all, we cite the following results:

Theorem $\mathbf{A}$ [1]. If $A \geqq B \geqq 0$, then for each $r \geqq 0$,

(i) $\left(B^{r} A^{p} B^{r}\right)^{1 / q} \geqq B^{(p+2 r) / q}$ and

(ii) $A^{(p+2 r) / q} \geqq\left(\bar{A}^{r} B^{p} A^{r}\right)^{1 / q}$

hold for each $p$ and $q$ such that $p \geqq 0, q \geqq 1$, and $(1+2 r) q \geqq p+2 r$.

Theorem B [2, 3]. If $A \geqq B \geqq 0$, then $A^{\alpha} \geqq B^{\alpha}$ for each $\alpha \in[0,1]$.

Lemma. Let $A$ and $B$ be invertible positive operators. For any real number $\lambda$,

$$
(B A B)^{\lambda}=B A^{1 / 2}\left(A^{1 / 2} B^{2} A^{1 / 2}\right)^{\lambda-1} A^{1 / 2} B \text {. }
$$

Proof of lemma. The proof follows from the known result that, for a continuous function $f(\lambda)$ on $(0, \infty), f\left(X^{*} X\right) X^{*} X=X^{*} f\left(X X^{*}\right) X$ for any operator $X$. For the sake of convenience, we give an easy proof here. We may assume that $A$ and $B$ are invertible because $T+\varepsilon \geqq \varepsilon>0$ for any positive operator $T$, so $T+\varepsilon$ is invertible. Let $B A^{1 / 2}=U \bar{H}$ be the polar decomposition of the invertible operator $B A^{1 / 2}$, where $U$ is the unitary and $H=\left|B A^{1 / 2}\right|$.

$$
\begin{aligned}
(B A B)^{\lambda} & =\left(U H^{2} U^{*}\right)^{\lambda}=U H^{2 \lambda} U^{*} \\
& =B A^{1 / 2} H^{-1} H^{2 \lambda} H^{-1} A^{1 / 2} B=B A^{1 / 2}\left(H^{2}\right)^{\lambda-1} A^{1 / 2} B \\
& =B A^{1 / 2}\left(A^{1 / 2} B^{2} A^{1 / 2}\right)^{\lambda-1} A^{1 / 2} B,
\end{aligned}
$$

so the proof is complete.

Proof of Theorem 1. First of all we prove the following (1) and (2). If $A \geqq B \geqq$ 0 , then for each $r \geqq 0$,

$$
B^{r} A^{p+s} B^{r} \geqq\left(B^{r} A^{p} B^{r}\right)^{(p+s+2 r) /(p+2 r)}
$$

and

$$
\left(A^{r} B^{p} A^{r}\right)^{(p+s+2 r) /(p+2 r)} \geqq A^{r} B^{p+s} A^{r}
$$

hold for each $p$ and $s$ such that $p \geqq 0$ and

$$
0 \leqq s \leqq \begin{cases}p+2 r & \text { if } 1 / 2 \geqq r \geqq 0, \\ p+1 & \text { if } r \geqq 1 / 2 .\end{cases}
$$

In order to prove (1) and (2), we prove the following (*) by the lemma:

$$
\left(B^{r} A^{p} B^{r}\right)^{(p+s+2 r) /(p+2 r)}=B^{r} A^{p / 2}\left(A^{p / 2} B^{2 r} A^{p / 2}\right)^{s /(p+2 r)} A^{p / 2} B^{r} \text {. }
$$

We first consider the case $1 / 2 \geqq r \geqq 0$. Then $A^{2 r} \geqq B^{2 r}$, by Theorem B and condition (3) of the hypothesis, ensures $s /(p+2 r) \in[0,1]$, so that $(*)$ and Theorem B yield

$$
\begin{aligned}
\left(B^{r} A^{p} B^{r}\right)^{(p+s+2 r) /(p+2 r)} & =B^{r} A^{p / 2}\left(A^{p / 2} B^{2 r} A^{p / 2}\right)^{s /(p+2 r)} A^{p / 2} B^{r} \\
& \leqq B^{r} A^{p / 2}\left(A^{p / 2} A^{2 r} A^{p / 2}\right)^{s /(p+2 r)} A^{p / 2} B^{r} \\
& =B^{r} A^{p+s} B^{r} .
\end{aligned}
$$


For the case $r \geqq 1 / 2$, in the term $\left(A^{p / 2} B^{2 r} A^{p / 2}\right)^{s /(p+2 r)}$ in the right-hand side of $(*), 2 r, p / 2$, and $(p+2 r) / s$ can surely be considered equivalent to $p, r$, and $q$ in Theorem $\mathrm{A}$. This is so because $(p+2 r) / s \geqq 1$ holds, since $p+2 r \geqq p+1 \geqq s \geqq 0$ by (3), and $\{1+2(p / 2)\}(p+2 r) / s \geqq 2 r+2(p / 2)$, also by (3). Therefore, by (ii) of Theorem A we have

$$
\left(A^{p / 2} B^{2 r} A^{p / 2}\right)^{s /(p+2 r)} \leqq A^{s} .
$$

By this inequality and $(*)$, we have

$$
\begin{aligned}
\left(B^{r} A^{p} B^{r}\right)^{(p+s+2 r) /(p+2 r)} & =B^{r} A^{p / 2}\left(A^{p / 2} B^{2 r} A^{p / 2}\right)^{s /(p+2 r)} A^{p / 2} B^{r} \\
& \leqq B^{r} A^{p / 2} A^{s} A^{p / 2} B^{r}=B^{r} A^{p+s} B^{r}
\end{aligned}
$$

hence we have (1).

By hypothesis, $B^{-1} \geqq A^{-1} \geqq 0$, since we may assume that $A$ and $B$ are invertible. Then, by (1),

$$
\left(A^{-r} B^{-p} A^{-r}\right)^{(p+s+2 r) /(p+2 r)} \leqq A^{-r} B^{-(p+s)} A^{-r}
$$

for the same $p, r$, and $s$ as in condition (3). Taking inverses gives (2). By using (1) and (2), we show (i) and (ii) as follows: The hypothesis $p \geqq 1$ of Theorem 1 ensures that $(1+2 r) /(p+s+2 r) \in[0,1]$ under the condition (3), so that by Theorem $\mathrm{B}$, taking $(1+2 r) /(p+s+2 r)$ as exponents of both sides of (1),

$$
F(p+s)=\left(B^{r} A^{p+s} B^{r}\right)^{(1+2 r) /(p+s+2 r)} \geqq\left(B^{r} A^{p} B^{r}\right)^{(1+2 r) /(p+2 r)}=F(p)
$$

holds for any $s$ satisfying (3). We are able to iterate this method whenever $p+s \geqq 1$, so we have (i) by repeating this method. By the same method as we used for (i), we can show (ii) by using (2), so the proof of Theorem 1 is complete.

Proof of Corollary 1. The proof follows by Theorem 1 if we put $r=1$ and $p_{1}=1, p_{2}=2, \ldots, p_{n-1}=p-1, p_{n}=p$.

Remark 1 . For the case $1 \geqq p \geqq 0$, Theorem A is obvious because $A^{p} \geqq B^{p}$ whenever $A \geqq B \geqq 0$ by Theorem B, so $\left(B^{r} A^{p} B^{r}\right)^{1 / q} \geqq\left(B^{r} B^{p} B^{r}\right)^{1 / q}=B^{(p+2 r) / q}$ for any $r \geqq 0$ and $q \geqq 1$ by Theorem B. Therefore the essential part of Theorem A asserts that $\left(B^{r} A^{p} B^{r}\right)^{(1+2 r) /(p+2 r)} \geqq B^{1+2 r}$ holds for each $p \geqq 1$ and $r \geqq 0$ whenever $A \geqq B \geqq 0$ because (i) of Theorem A, for values $q$ larger than $(p+2 r) /(1+2 r)$, follows by Theorem B. Consequently we remark that (i) and (ii) of Theorem 1 can surely be considered extensions of Theorem A.

\section{RELATED COUNTEREXAMPLES}

Counterexample 1. In Theorem 1 , the condition $p \geqq 1$ is essential. We cite a counterexample to Theorem 1 for $0<p<1$. Put $r=0.1, p_{1}=0.99$, and $p_{2}=0.1$. Take $B$ and $C$ as

$$
B=\left(\begin{array}{cc}
0 & -1 \\
-1 & 13
\end{array}\right)^{2} \geqq 0, \quad C=\left(\begin{array}{cc}
14 & 13 \\
13 & 11
\end{array}\right)^{2} \geqq 0 .
$$


Then $A=B+C \geqq B \geqq 0$, but computation shows that

$$
\begin{aligned}
D & =\left(B^{r} A^{p_{1}} B^{r}\right)^{(1+2 r) /\left(p_{1}+2 r\right)}-\left(B^{r} A^{p_{2}} B^{r}\right)^{(1+2 r) /\left(p_{2}+2 r\right)} \\
& =\left(\begin{array}{cc}
103.43841 \ldots & 253.74356 \ldots \\
253.74356 \ldots & 604.34393
\end{array}\right) .
\end{aligned}
$$

The eigenvalues of $D$ are $-2.63705 \ldots$ and $710.41940 \ldots$. Hence

$$
\left(B^{r} A^{p_{1}} B^{r}\right)^{(1+2 r) /\left(p_{1}+2 r\right)} \supsetneqq\left(B^{r} A^{p_{2}} B^{r}\right)^{(1+2 r) /\left(p_{2}+2 r\right)},
$$

even if $1>p_{1}>p_{2}>0$.

Counterexample 2. In (1), the condition (3) is essential. We cite a counterexample to (1) for $r, p$, and $s$ without this condition. From (3), $0 \leqq s \leqq p+1$ if $r \geqq 1 / 2$. Put $r=0.510>1 / 2, p=0.49$, and $s=1.50$. Then $0 \leqq s \$ p+1$. Take $B$ and $C$ as

$$
B=\left(\begin{array}{cc}
10 & 1 \\
1 & 2
\end{array}\right)^{2} \geqq 0, \quad C=\left(\begin{array}{ll}
0 & 0 \\
0 & 5
\end{array}\right)^{2} \geqq 0 .
$$

Then $A=B+C \geqq B \geqq 0$, but computation shows that

$$
\begin{aligned}
D & =B^{r} A^{p+s} B^{r}-\left(B^{r} A^{p} B^{r}\right)^{(p+s+2 r) /(p+2 r)} \\
& =\left(\begin{array}{cc}
4616.68997 \ldots & 3965.23361 \ldots \\
3965.23361 \ldots & 3393.69398 \ldots
\end{array}\right) .
\end{aligned}
$$

The eigenvalues of $D$ are $-6.91562 \ldots$ and $8017.29957 \ldots$. Consequently, $B^{r} A^{p+s} B^{r} \nsucceq\left(B^{r} A^{p} B^{r}\right)^{(p+s+2 r) /(p+2 r)}$.

Counterexample 3. We can also give a counterexample to (1) for $r, p$, and $s$ without condition (3), $0 \leqq s \leqq p+2 r$ if $1 / 2 \geqq r \geqq 0$. Put $r=0.45<1 / 2$, $p=0.5$, and $s=1.45$. Then $0 \leqq s \$ p+2 r$. Take $B$ and $C$ as

$$
B=\left(\begin{array}{cc}
-1 & 2 \\
2 & 5
\end{array}\right)^{2} \quad C=\left(\begin{array}{cc}
15 & 0 \\
0 & 0
\end{array}\right)^{2} .
$$

Then $A=B+C \geqq B \geqq 0$, but computation shows that

$$
\begin{aligned}
D & =B^{r} A^{p+s} B^{r}-\left(B^{r} A^{p} B^{r}\right)^{(p+s+2 r) /(p+2 r)} \\
& =\left(\begin{array}{cc}
129850.96526 \ldots & 70741.78325 \ldots \\
70741.78325 \ldots & 38537.76032 \ldots
\end{array}\right) .
\end{aligned}
$$

The eigenvalues of $D$ are $-1.39272 \ldots$ and $168390.11831 \ldots$ Hence $B^{r} A^{p+s} B^{r} \nsucceq\left(B^{r} A^{p} B^{r}\right)^{(p+s+2 r) /(p+2 r)}$.

\section{ACKNOWLEDGMENT}

The author would like to express his sincere thanks to the referee for useful comments about the first version. 
Addendum. We have the following further extensions:

Theorem 2. If $A \geqq B \geqq 0$, then for a fixed $t \geqq 0$ and $r \geqq 0$,

(i) $F_{t}(p)=\left(B^{r} A^{p} B^{r}\right)^{(t+2 r) /(p+2 r)}$ is an increasing function of $p$ for $p \geqq t \geqq$ 0 .

(ii) $G_{t}(p)=\left(A^{r} B^{p} A^{r}\right)^{(t+2 r) /(p+2 r)}$ is a decreasing function of $p$ for $p \geqq t \geqq$ 0 .

Theorem 3. If $A \geqq B \geqq 0$, then for a fixed $t \geqq 0$ and $r \geqq 0, F(p, r)=$ $\lim _{c \downarrow 0} B_{c}^{-r}\left(B_{c}^{r} A_{c}^{p} B_{c}^{r}\right)^{(t+2 r) /(p+2 r)} B_{c}^{-r}$ is an increasing function of both $p$ and $r$ for $p \geqq t \geqq 0$, where $X_{c}=X+c I$.

Corollary 2 (Ando).If $A \geqq B \geqq 0$, then for $p \geqq 0$,

$$
B^{-p} \# A^{p}=\lim _{c \downarrow 0} B_{c}^{-p / 2}\left(B_{c}^{p / 2} A_{c}^{p} B_{c}^{p / 2}\right)^{1 / 2} B_{c}^{-p / 2}
$$

is an increasing function of $p$, where $X_{c}=X+c I$.

Proof of Theorem 2. By almost the same method as the one in Theorem 1, we can show Theorem 2, so we omit its proof.

Proof of Theorem 3. We may assume that $A$ and $B$ are invertible. First of all, we show the following:

$$
\left(A^{p / 2} B^{2 r} A^{p / 2}\right)^{(t-p) /(2 r+p)} \geqq\left(A^{p / 2} B^{2 s} A^{p / 2}\right)^{(t-p) /(2 s+p)} \quad \text { for } r \geqq s \geqq 0 .
$$

By (ii) of Theorem 2, we have

$$
\left(A^{p / 2} B^{2 r} A^{p / 2}\right)^{\left(t_{1}+p\right) /(2 r+p)} \leqq\left(A^{p / 2} B^{2 s} A^{p / 2}\right)^{\left(t_{1}+p\right) /(2 s+p)} \quad \text { for } 2 r \geqq 2 s \geqq t_{1} \geqq 0 .
$$

Put $k=(p-t) /\left(p+t_{1}\right)$. Then $k \in[0,1]$ since $p \geqq t \geqq 0$ and $t_{1} \geqq 0$. By Theorem B, taking $k$ as exponents of both sides of (5) and moreover taking inverses of these both sides, we have (4).

$$
\begin{aligned}
F(p, r) & =B^{-r}\left(B^{r} A^{p} B^{r}\right)^{(t+2 r) /(p+2 r)} B^{-r} \\
& =A^{p / 2}\left(A^{p / 2} B^{2 r} A^{p / 2}\right)^{(t-p) /(p+2 r)} A^{p / 2} \quad \text { by Lemma } \\
& \geqq A^{p / 2}\left(A^{p / 2} B^{2 s} A^{p / 2}\right)^{(t-p) / p+2 s)} A^{p / 2} \quad \text { for } r \geqq s \geqq 0 \text { by }(4) \\
& =B^{-s}\left(B^{s} A^{p} B^{s}\right)^{(t+2 s) /(p+2 s)} B^{-s} \quad \text { by Lemma } \\
& =F(p, s) \text { for } r \geqq s \geqq 0,
\end{aligned}
$$

so the proof of Theorem 3 is complete since $F(p, r)$ is an increasing function of $p$ by Theorem 2 .

Proof of Corollary 2. Put $r=p / 2$ and $t=0$ in Theorem 3.

Mean theoretic proof of Theorem 3 will appear in the following paper: M. Fujii, T. Furuta, and E. Kamei, Operator functions associated with Furuta's inequality, preprint. 


\section{REFERENCES}

1. T. Furuta, $A \geqq B \geqq 0$ assures $\left(B^{r} A^{p} B^{r}\right)^{1 / q} \geqq B^{(p+2 r) / q}$ for $r \geqq 0, p \geqq 0, q \geqq 1$ with $(1+2 r) q \geqq p+2 r$, Proc. Amer. Math. Soc. 101 (1987), 85-88.

2. K. Löwner, Über monotone Matrixfunktionen, Math. Z. 38 (1934), 177-216.

3. G. K. Pedersen, Some operator monotone functions, Proc. Amer. Math. Soc. 36 (1972), 309-310.

Department of Mathematics, Faculty of Science, Hirosaki University, Bunkyo-cho 3, HIROSAKI, AOMORI 036 JAPAN 